\title{
Wireless distance measurement by means of ultra-wideband chaotic radio pulses
}

\author{
Elena Efremova ${ }^{1,2,}$, Alexander Dmitriev ${ }^{1,2}$, Lev Kuzmin ${ }^{1,2}$, and Manvel Petrosyan ${ }^{1,2}$ \\ ${ }^{1}$ Kotelnikov Institute of Radio Engineering and Electronics of RAS, 125009, Moscow, Russia \\ ${ }^{2}$ Moscow Institute of Physics and Technology (National Research University), 141701, Dolgoprudny, \\ Russia
}

\begin{abstract}
A method for wireless distance measurement using ultrawideband chaotic radio pulses based on statistical analysis is proposed. Experimental results are discussed.
\end{abstract}

\section{Introduction}

Indoor localization in the absence of global positioning services is an actual area especially in the era of Internet of Things (IoT), machine-to-machine interaction (M2M), robotics, etc.

To date, a number of indoor positioning systems is proposed and realized. They use different wireless technologies, such as WiFi, Bluetooth, BLE, ZigBee, UWB, acoustics and so on. The main approaches to distance measurement in wireless systems are based on estimation of signal strength (RSSI), time of flight (TOA, TDOA, RTOF, etc.), or phase [1,2].

Depending on the technology, measurement method and post-processing tools, the distance accuracy is $15 \mathrm{~cm}$ to several meters. The best accuracy is achieved in systems based on ultra-wideband (UWB) ultra-short pulses. These systems use time of flight estimation, to measure distance. However, such systems have the most complex hardware.

UWB chaotic radio pulses are one of the UWB signal types [3, 4]. They are practically immune to multipath fading in wireless channels [5]. As is shown in the experiments [6], UWB signals provide smooth dependence of signal power on the distance according to power law $1 / d^{n}$. As for narrow band signals (e.g., used in ZigBee), it is not so [7].

In the narrowband systems, multipath propagation leads to high variations of the measured signal power in the receiver, which results in large errors of distance estimation.

This multipath immunity gives a reason for distance measurement by means of pulse power estimation in the receiver based on the power attenuation law.

From a practical point of view, another reason for the use of chaotic radio pulses for distance measurement is that they are an optional solution in communication systems of IEEE 802.15.4 standard and one of the main solutions of IEEE 802.15.6 standard.

In the existing equipment $[8,9]$, chaotic radio pulses are used to transmit information, so it is practically interesting to create a method for distance measurement, which would be a part of the process of wireless data exchange between the communication devices.

Here, a possibility of distance measurement with UWB chaotic radio pulses, based on the measurement of relative power of the signal at the receiver input using a statistical analysis of its characteristics is investigated experimentally.

* Corresponding author: efremova@cplire.ru 


\section{Method}

The idea of the method is to form and to emit a train of chaotic radio pulses by a transmitter, to detect them by a receiver, to estimate the pulse power and to calculate the number of the pulses whose power exceeds a preassigned level. Based on this information and on the channel power attenuation law, the distance between the transmitter and receiver is evaluated.

Let $P_{\mathrm{d}} \sim 1 / d^{n}$ be the power attenuation rule at distance $d$ between transmitter and receiver, where $n$ is the attenuation rate in a real wireless channel. Then $P_{\mathrm{d}}$ can be calculated as

$$
P_{d}=P_{0}+10 n \lg \left(d / d_{0}\right),
$$

where $P_{0}$ is power attenuation at distance $d_{0}[10]$.

In the experiment, three values can be obtained: $P_{\mathrm{d}}, P_{0}$, and $d_{0} . P_{\mathrm{d}}$ and $P_{0}$ can be measured with a log-detector that forms the output signal with amplitude $A_{\mathrm{d}}$ proportional to the input signal power $P_{\mathrm{d}} . d_{0}$ is determined simultaneously with $P_{0}$.

Power pathloss $P_{\text {add }}=10 n \lg \left(d / d_{0}\right)$ is defined as difference $10 n \lg (\mathrm{d} / \mathrm{d} 0)=P_{\mathrm{d}}-P_{0}$ by means of comparing amplitude $A_{\mathrm{d}}$ of the pulses with power $P_{\mathrm{d}}$ at the input of log-detector with the amplitude $A_{0}$ of the signal with power $P_{0}$ at distance $d_{0}$ from the emitter. Using the input signal power-to-output voltage dependence [11] we can obtain:

$$
P_{\mathrm{d}}-P_{0}=\left(A_{\mathrm{d}}-A_{0}\right) / h,
$$

where $h$ is the slope of the detector characteristics.

Distance $d$ is calculated as

$$
\left.d=d_{0} 10_{\mathrm{d}}^{\left(P_{0}-P_{0}\right) /(10 n)}=d_{0} 10_{\mathrm{d}}^{(A}-A_{0}\right) /(10 n h)
$$

In the free space $n=2$, in a real wireless channel with line of sight $n<2$, in a no-line-ofsight channel $n>3$ [12].

Amplitude $A_{\mathrm{d}}$ is measured by means of comparing it with some variable threshold level $A_{T}$. In more details, for a fixed threshold level $A_{T}$ the number of exceeding pulses is counted; and then threshold $A_{T}$ is varied, in order to find the level at which the detector stops "feeling" the pulses.

Due to chaotic nature of the signal, the power of UWB chaotic pulses varies from pulse to pulse. Distributions of the pulse envelope amplitudes in the detector measured in the experiment at different distances between emitter and receiver is shown in Fig. 1.

Note that the width of the pulse amplitude distribution for different distances $d$ remains approximately the same. Since the distance is calculated from the difference of amplitudes $\left(A_{\mathrm{d}}-A_{0}\right)$, this allows us to fix the error level for a reference distance $d_{0}$ and distance $d$ at the reception point. By means of estimating threshold level $A_{\mathrm{T}}$ for these distances at a fixed error level it is possible to calculate distance $d$.

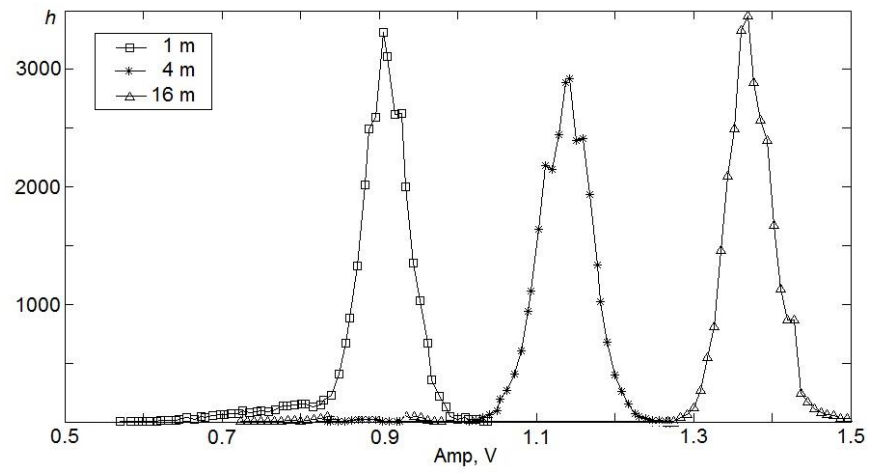

Fig. 1. Distribution of amplitudes Amp of the envelope of chaotic radio pulses in the receiver at various distances $d$ between the emitter and the receiver. Squares $-1 \mathrm{~m}$, stars $-4 \mathrm{~m}$, triangles $-16 \mathrm{~m}$. 


\section{Experiment}

To confirm the idea, an experiment was carried out. Experimental setup consists of two UWB chaotic transceivers [9] that play the role of the source and the receiver of chaotic radio pulses. Frequency range of the chaotic signal emitted by the source is $F=3-5 \mathrm{GHz}$. The devices were located in direct line of sight. The receiver position was fixed, the transmitter was moved by $0.25-0.5 \mathrm{~m}$ steps along a straight line. The scheme of the experiment is shown in Fig. 2.

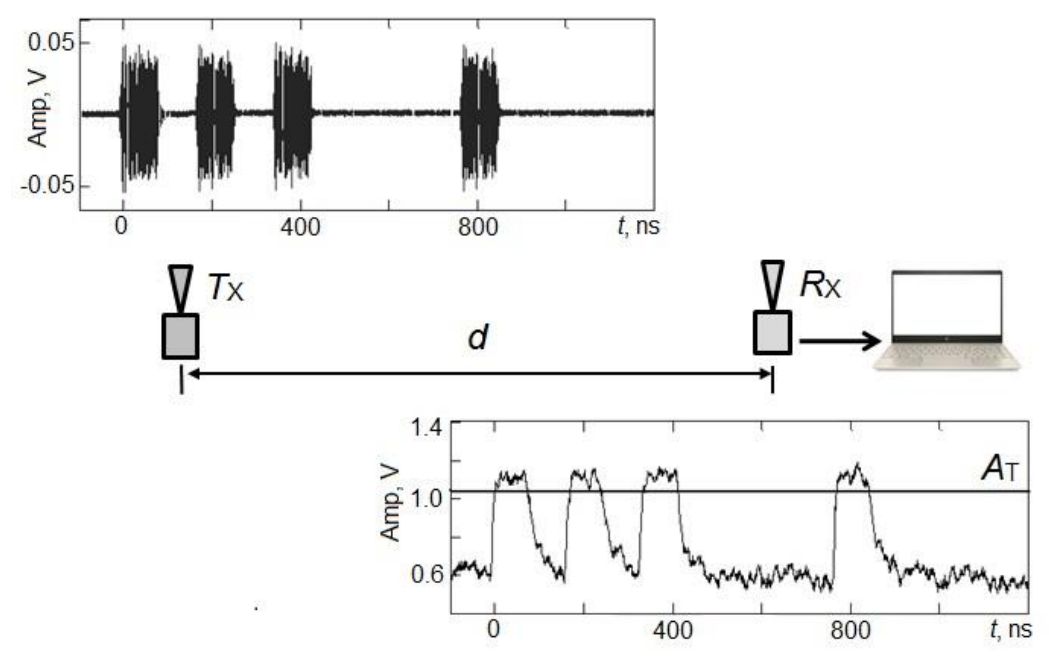

Fig. 2. Scheme of the experiment.

Transmitter Tx forms chaotic radio pulse packets supplemented with service information (number of pulses, check sum) necessary to control the received packet integrity.

In receiver $\mathrm{Rx}$ the signal is amplified, is passed through log-detector [11] and is compared with threshold. If envelope amplitude is higher than the threshold, then the pulse is considered received; otherwise, not.

The number of pulses that meet the condition is counted. The process is equivalent to the scheme of packet reception during data exchange between transceivers [8].

For a given distance $d$ between signal emitter and receiver the fraction of packets $P_{B}(d)$, received with an error (PER - packet error ratio) is calculated. For every $d$, the threshold level $A_{d}$ is varied so as to obtain a preassigned level $P_{B}(d)$ of packet error. The result of the experiment is the dependence of threshold voltage $A_{d}$ on distance $d$ at a given level of $P_{B}(d)$.

In the experiment, the threshold value $T_{\mathrm{d}}$ can be set at one of 256 positions (provided by DAC) corresponding to varying $A_{\mathrm{d}}$ in the range $[0,3.3] \mathrm{V}$. Thus, the threshold value is set with precision $k=3.3 / 255=12.9 \mathrm{mV}$. Since the detector slope is $h=-22 \mathrm{mV} / \mathrm{dB}$ [11], one threshold step corresponds to a change of the input power by $S=k / h \approx-0.59 \mathrm{~dB}$.

As follows from expression (3), the threshold to distance conversion is given by

$$
d=d_{0} 10_{\mathrm{d}}^{(T-T) k /(10 n h)}
$$

where $T_{0}, T_{\mathrm{d}}$ are threshold values at the reference point (on the distance $d_{0}$ ) and on the distance $d$ (at given error level), respectively, $k$ is increment of the amplitude (Volts) corresponding to one threshold step $\left(A_{T}=T_{\mathrm{d}} k\right)$.

Taking into account theoretical dependence (4), where $d_{0}=1 \mathrm{~m}$, after conversion to logarithms, we have: 


$$
\left(T_{d}-T_{0}\right) S=10 n \lg d
$$

With the measured values $10 \lg d$ put along $\mathrm{X}$-axis and the values $\left(T_{\mathrm{d}}-T_{0}\right) S$ along $\mathrm{Y}$-axis, where $S=-0.59 \mathrm{~dB}$, the dependence $10 \lg d$ on $\left(T_{d}-T_{0}\right) S$ must be linear with the slope $n$.

The experiments were set on two locations in Kotelnikov IRE RAS: in the conference hall (A) and in an office (B). In Figs. 3 and 4 the results for the conference hall are presented.

The dependence of threshold value $T_{\mathrm{d}}$ on distance $d$ was measured. The threshold value was taken at $\mathrm{PER} \geq 95 \%$.

Five measurements were made in each experimental point.

The first measurement was used to determine attenuation rate $n$. The rate $n$ was determined using mean square method as the slope of the straight line approximating the experimental dependence on $\left(10 \lg d,\left(T_{\mathrm{d}}-T_{0}\right) S\right)$ plane (fig. 3). In the figure, the dependence is well approximated by a straight line with $n=1.76$. This value was used to evaluate the distances between the emitter and receiver.

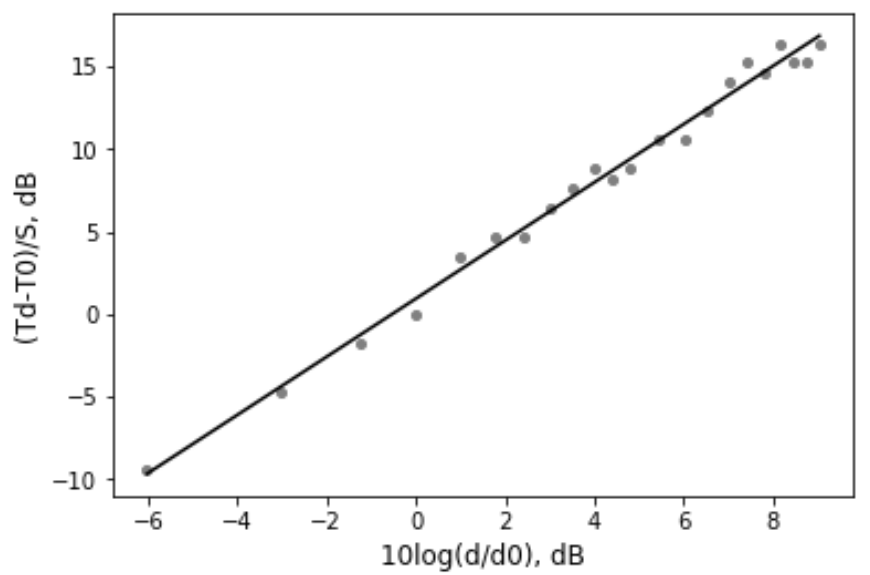

Fig. 3. Threshold difference $\left(T_{d}-T_{0}\right) S$ as a function of $10 \lg \left(d / d_{0}\right)$ in experiment.

In Fig. 4 the measurement results as functions of the actual distance are depicted, including the measured distance estimate, absolute and relative errors. For each real distance, the mean value and standard deviation of the measured parameter (over 5 measurements) are shown.

The average relative error for conference hall $\mathrm{A}$ at distances up to $8 \mathrm{~m}$ was $15 \%$. For room $\mathrm{B}$ the results are similar, the average relative error at distances up to $5 \mathrm{~m}$ was $13 \%$.

\section{Conclusions}

A method of distance measurement in wireless channel based on statistical analysis is proposed and experimentally tested. The method is using UWB chaotic radio pulses. Its results are comparable with modern positioning technologies, whereas its technical implementation is much simpler and does not require additional capabilities of the existing equipment above those related with the reception and processing of UWB signal during wireless information transmission.

This study was supported by a state order for the Kotelnikov Institute of Radio Engineering and Electronics, Russian Academy of Sciences. 


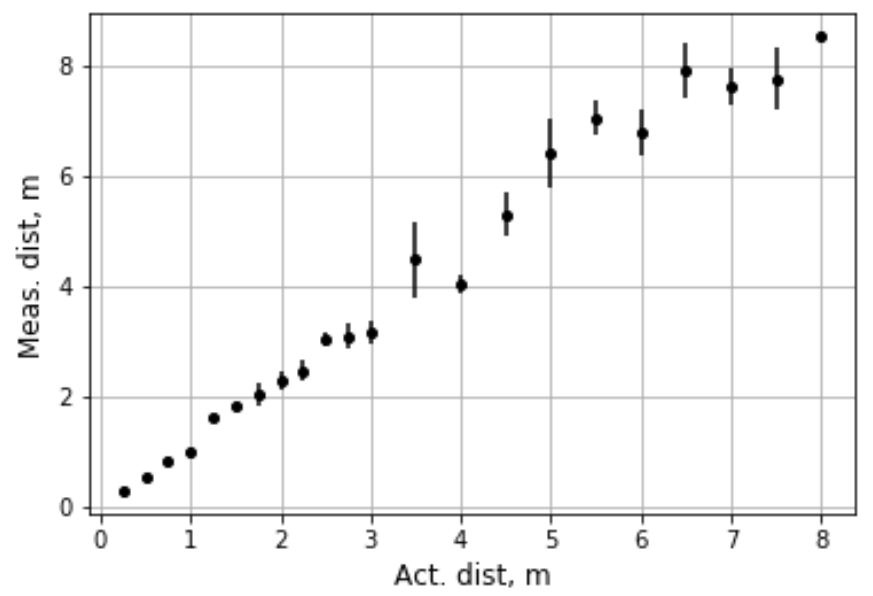

(a)

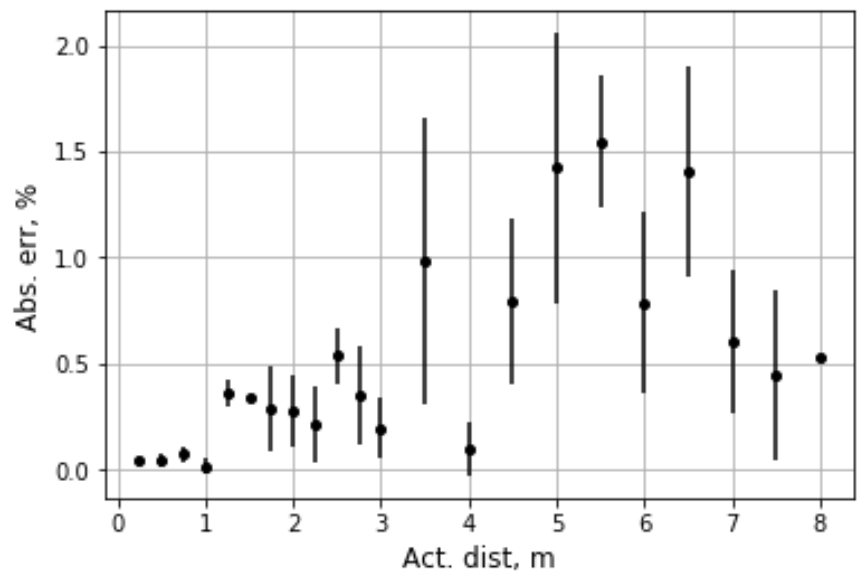

(b)

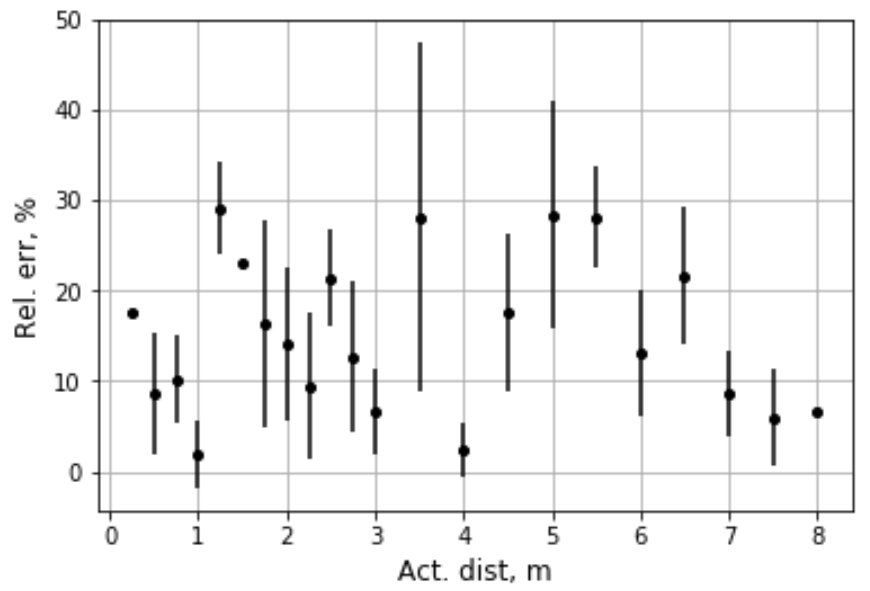

(c)

Fig. 4. Measurement results as functions of the actual distance. (a) measured distance estimate; (b) absolute measurement error; (c) relative error. 


\section{References}

1. F. Zafari, A. Gkelias, and K. K. Leung, arXiv: 1709.01015v3 (1709)

2. A. Alarifi, A. Al-Salman, M. Alsaleh, A. Alnafessah, S. Al-Hadhrami, M. A. AlAmmar, and H. S. Al-Khalifa, Sensors 16, 707 (2016)

3. A. S. Dmitriev, E. V. Efremova, A. I. Panas, and N. A. Maksimov, Generation of Chaos (Tekhnosfera, Moscow, 2012) [in Russian]

4. A. S. Dmitriev and E. V. Efremova, Tech. Phys. Lett. 42, 42 (2016)

5. Yu. V. Andreev, A. S. Dmitriev, and V. A. Lazarev, in Proceedings of the 5th AllRussia Armand's Readings, Murom, p. 211 (2015)

6. Yu. V. Gulyaev, A. S. Dmitriev, V. A. Lazarev, T. I. Mokhseni, and M. G. Popov, J. Commun. Technol. Electron. 61, 894 (2016)

7. A. V. Ponikar, O. V. Evseev, V. E. Antsiperov, and G. K. Mansurov, in Proceedings of the 4th All-Russia Conference on Radiolocation and Radio Communication (IRE RAN, Moscow, 2010), p. 914.

8. A. S. Dmitriev, E. V. Efremova, A. V. Kletsov, L. V. Kuz'min, A. M. Laktyushkin, and V. Yu. Yurkin, J. Commun. Technol. Electron. 53, 1206 (2008)

9. A. S. Dmitriev, M. Yu. Gerasimov, V. V. Itskov, V. A. Lazarev, M. G. Popov, and A. I. Ryzhov, J. Commun. Technol. Electron. 62, 380 (2017)

10. B. Sklar, Digital Communications: Fundamentals and Applications (Prentice Hall, 2017)

11. Analog Device Data Sheet for AD8317 $1 \mathrm{MHz}-10 \mathrm{GHz} 50 \mathrm{~dB}$ High Precision Logarithmic Detector. http://www.analog.com.

12. A. F. Molisch, Proc. IEEE 97, 353 (2009) 